\title{
SPACELIKE JORDAN OSSERMAN ALGEBRAIC CURVATURE TENSORS IN THE HIGHER SIGNATURE SETTING
}

\author{
PETER B. GILKEY AND RAINA IVANOVA \\ Mathematics Department, University of Oregon, Eugene Or 97403 USA \\ email: gilkey@darkwing.uoregon.edu, ivanovar@hopf.uoregon.edu \\ Dedicated to Professor Navaira
}

\begin{abstract}
Let $R$ be an algebraic curvature tensor on a vector space of signature $(p, q)$ defining a spacelike Jordan Osserman Jacobi operator $\mathcal{J}_{R}$. We show that the eigenvalues of $\mathcal{J}_{R}$ are real and that $\mathcal{J}_{R}$ is diagonalizable if $p<q$.

Subject Classification: 53B20
\end{abstract}

\section{Introduction}

Let $V$ be a vector space that is equipped with an inner product $(\cdot, \cdot)$ of signature $(p, q)$. The inner product is said to be Riemannian if $p=0$, Lorentzian if $p=1$, and balanced (or neutral) if $p=q$. Let $R$ be an algebraic curvature tensor on $V$; i.e. $R \in \otimes^{4} V^{*}$ satisfies the curvature symmetries of the Riemann curvature tensor:

$$
\begin{aligned}
& R(x, y, z, w)=R(z, w, x, y)=-R(y, x, z, w), \text { and } \\
& R(x, y, z, w)+R(y, z, x, w)+R(z, x, y, w)=0 .
\end{aligned}
$$

The associated Jacobi operator $\mathcal{J}_{R}$ is the self-adjoint linear map of $V$ which is defined by the identity:

$$
\left(\mathcal{J}_{R}(x) y, z\right)=R(y, x, x, z) .
$$

We say that an algebraic curvature tensor $R$ is spacelike Osserman if the eigenvalues of $\mathcal{J}_{R}$ are constant on the pseudo-sphere $S^{+}(V)$ of unit spacelike vectors. We say that $R$ is spacelike Jordan Osserman if the Jordan normal form of $\mathcal{J}_{R}$ is constant on $S^{+}(V)$. In the Riemannian context, these two notions are equivalent. However, in higher signature setting, the eigenvalue structure does not determine the conjugacy class, so we shall work with Jordan normal form rather than with the eigenvalues alone.

The investigation of spacelike Jordan Osserman tensors is motivated by geometric considerations. Let $(M, g)$ be a pseudo-Riemannian manifold of signature $(p, q)$. If ${ }^{g} R$ is the Riemann curvature tensor of the Levi-Civita connection, then ${ }^{g} R_{P}$ is an algebraic curvature tensor on the tangent space

P147-GilkeyIvanova-v3a: submitted to World Scientific on October 26, 20181 
$T_{P} M$ for every $P$ in $M$. On the other hand, every algebraic curvature tensor is geometrically realizable. We say that $(M, g)$ is spacelike Jordan Osserman if the Jordan normal form of $\mathcal{J}_{g_{R}}$ is constant on the pseudo-sphere bundle $S^{+}(M, g)$ of unit spacelike tangent vectors.

Let $(M, g)$ be Riemannian. Then $(M, g)$ is said to be a local 2 point homogeneous space if the local isometries of $(M, g)$ act transitively on $S^{+}(M, g)$; such a manifold is either flat or locally isometric to a rank 1 symmetric space. If $(M, g)$ is a local 2 point homogeneous space, then the Jacobi operator $\mathcal{J}_{R}$ has constant Jordan normal form on $S^{+}(M, g)$ and hence $(M, g)$ is spacelike Jordan Osserman. Osserman 13 wondered if the converse held. Chit showed this was true if $m=4$, if $m \equiv 1 \bmod 2$, or if $m \equiv 2 \bmod 4$; an important ingredient in Chi's work was the classification of the spacelike Jordan Osserman Riemannian algebraic cyrvature tensors in these dimensions. There are other partial results available 11 , but the question is still open in the Riemannian context.

It has been shownl 6 that any Lorentzian spacelike Jordan Osserman algebraic curvature tensor has constant sectional curvature. Thus classification is complete in this context. Finally, it is knowne 3 . that there exist balanced spacelike Jordan Osserman pseudo-Riemannian manifolds which are not even locally homogeneous.

We shall work in the algebraic category henceforth. If $W$ is an auxiliary vector space and if $A$ is a linear map of $V$, then we define the stabilization

$$
A \oplus 0:=\left(\begin{array}{cc}
A & 0 \\
0 & 0
\end{array}\right) \text { on } V \oplus W .
$$

The Jordan normal form of a spacelike Jordan Osserman algebraic curvature can be arbitrarily complicated in the balanced settingete

Theorem 1.1 Let $J$ be a $r \times r$ real matrix and let $q \equiv 0 \bmod 2^{r}$. If $V$ is a vector space of neutral signature $(q, q)$, then there exists an algebraic curvature tensor $R$ on $V$ so that $\mathcal{J}_{R}(x)$ is conjugate to $J \oplus 0$ for every $x \in S^{+}(V)$.

However, the situation is very different if $p<q$, i.e. if the spacelike directions in a certain sense dominate the timelike directions. We consider this case and show that the geometry defined by such a tensor is much more rigid. The main result of this paper is:

Theorem 1.2 Let $R$ be a spacelike Jordan Osserman algebraic curvature tensor on a vector space $V$ of signature $(p, q)$, where $p<q$. Then $\mathcal{J}_{R}(x)$ is diagonalizable for any $x \in S^{+}(V)$.

Here is a brief outline of the paper. In $\{2$, we review certain results concerning self-adjoint maps in the indefinite setting. In $\delta$, we establish

P147-GilkeyIvanova-v3a: submitted to World Scientific on October 26, 20182 
several technical results concerning vector bundles over projective spaces. In \$4, we use the results of the previous sections to prove Theorem 1.2 .

\section{Results from Linear Algebra}

Let $\Re(\lambda)$ and $\Im(\lambda)$ be the real and imaginary parts of a complex number $\lambda$. If $J$ is a linear map of a real vector space $V$ of dimension $m$, then let $J_{\lambda}$ be the real operator on $V$ defined by:

$$
J_{\lambda}:= \begin{cases}J-\lambda \cdot \mathrm{Id} & \text { if } \quad \lambda \in \mathbb{R} \\ (J-\lambda \cdot \mathrm{Id})(J-\bar{\lambda} \cdot \mathrm{Id}) & \text { if } \quad \lambda \in \mathbb{C}-\mathbb{R} .\end{cases}
$$

We define the generalized eigenspaces by setting

$$
E_{\lambda}=E_{\lambda}^{J}:=\operatorname{ker}\left\{J_{\lambda}^{m}\right\} .
$$

Lemma 2.1 Let $V$ be a vector space of signature $(p, q)$ and let $J$ be a selfadjoint linear map of $V$. Then $V$ can be decomposed as an orthogonal direct sum $V=\oplus_{\Im(\lambda) \geq 0} E_{\lambda}$. Furthermore, the induced metrics on the generalized eigenspaces $E_{\lambda}$ are non-degenerate.

Proof: Let $\lambda$ and $\mu$ be complex numbers with $\lambda \neq \mu$ and $\lambda \neq \bar{\mu}$. Since $J_{\lambda}^{m}$ is self-adjoint and vanishes on $E_{\lambda}$, we have

$$
0=\left(J_{\lambda}^{m} x_{\lambda}, x_{\mu}\right)=\left(x_{\lambda}, J_{\lambda}^{m} x_{\mu}\right) \text { for } x_{\lambda} \in E_{\lambda} \text { and } x_{\mu} \in E_{\mu} .
$$

Since $J$ commutes with $J_{\mu}, J$ preserves $E_{\mu}$. Since the eigenvalues of $J$ on $E_{\mu}$ are $\mu$ and $\bar{\mu}$, the linear maps $J-\lambda \cdot \operatorname{Id}, J-\bar{\lambda} \cdot \operatorname{Id}$, and hence $J_{\lambda}$ are isomorphisms of $E_{\mu}$; thus $J_{\lambda}^{m}\left(E_{\mu}\right)=E_{\mu}$. It now follows that

$$
E_{\lambda} \perp E_{\mu} \text { and } E_{\lambda} \cap E_{\mu}=\{0\} .
$$

Let $V^{\mathbb{C}}:=V \otimes \mathbb{C}$ be the complexification of $V$. We extend $J$ to $V^{\mathbb{C}}$ to be complex linear and set $E_{\lambda}^{\mathbb{C}}:=\operatorname{ker}\left\{(J-\lambda)^{m}\right\}$. A complex vector space may be decomposed as the direct sum of the generalized complex eigenspaces defined by a linear transformation. Consequently,

$$
V^{\mathbb{C}}=\oplus_{\lambda} E_{\lambda}^{\mathbb{C}} .
$$

As $E_{\lambda}^{\mathbb{C}} \oplus E_{\bar{\lambda}}^{\mathbb{C}}=E_{\lambda} \otimes \mathbb{C}, V=\oplus_{\Im(\lambda) \geq 0} E_{\lambda}$. By display (2.3), the direct sum given in equation (2.4) is orthogonal; thus, the induced metric on each $E_{\lambda}$ is non-degenerate.

P147-GilkeyIvanova-v3a: submitted to World Scientific on October 26, 20183 
We recall a few notions from bundle theory which we will use in what follows. Let $\rho: E \rightarrow M$ be a vector bundle over a smooth manifold $M$. The fibers $E_{P}:=\rho^{-1}(P)$ are real vector spaces which vary smoothly with the point $P \in M$. A non-degenerate fiber metric on $E$ is a collection of non-degenerate inner products on each fiber which vary smoothly on $M$. A bundle morphism $\psi$ of $E$ is a collection of smooth linear maps $\psi_{P}$ of the fibers $E_{P}$ which vary smoothly with $P$. We say $\psi$ is invertible if each $\psi_{P}$ is invertible. We say $\psi$ is self-adjoint if each $\psi_{P}$ is self-adjoint.

Let $V$ be a vector space with a non-degenerate inner product. We can decompose $V$ as a direct sum $V^{+} \oplus V^{-}$of complementary orthogonal subspaces, where $V^{+}$is a maximal spacelike subspace and $V^{-}$is a maximal timelike subspace. There is a similar decomposition possible in the vector bundle setting:

Lemma 2.2 Let $E$ be a vector bundle over a smooth manifold $M$ which is equipped with a non-degenerate fiber metric. Then we can decompose $E$ as a direct sum $E^{+} \oplus E^{-}$of complementary orthogonal subbundles, where $E^{+}$is a maximal spacelike subbundle and $E^{-}$is a maximal timelike subbundle.

Proof: As noted above, we can decompose each individual fiber as an orthogonal direct sum of a maximal spacelike and a maximal timelike subspace. The main technical difficulty is to make the decompositions vary smoothly with $P \in M$. We can use a partition of unity to put a positive definite inner product $(\cdot, \cdot)_{e}$ on $E$. Define a bundle morphism $\psi$ of $E$ by setting $(v, w)=(\psi v, w)_{e}$. Since each linear map $\psi_{P}$ is self-adjoint with respect to the positive definite inner product $(\cdot, \cdot)_{e}$ on each fiber $E_{P}, \psi_{P}$ is diagonalizable and has only real eigenvalues. As the original inner product $(\cdot, \cdot)$ is non-degenerate, each $\psi_{P}$ is invertable. Let $E_{\lambda}\left(\psi_{P}\right) \subset E_{P}$ be the eigenspaces of $\psi_{P}$ on $E_{P}$. We define:

$$
E_{P}^{-}:=\oplus_{\lambda<0} E_{\lambda}\left(\psi_{P}\right) \text { and } E_{P}^{+}:=\oplus_{\lambda>0} E_{\lambda}\left(\psi_{P}\right) .
$$

By Lemma 2.1, the subspaces $E_{P}^{+}$and $E_{P}^{-}$are orthogonal and complementary. Since $\psi_{P}$ is invertible, the fibers $E_{P}^{+}$and $E_{P}^{-}$have constant rank and define smooth subbundles of $E$.

We continue our preparation for the proof of Theorem 1.2 by studying vector bundles equipped with non-degenerate fiber metrics and self-adjoint bundle morphisms which have constant Jordan normal form:

Lemma 2.3 Let $E$ be a vector bundle over a smooth manifold $M$ which is equipped with a non-degenerate fiber metric. Let $J$ be a self-adjoint bundle morphism of $E$ which has constant Jordan normal form. Let $\lambda$ be an eigenvalue of $J$. If $J_{\lambda} \neq 0$ on $E_{\lambda}$, choose $i \geq 1$ maximal so $J_{\lambda}^{i}\left(E_{\lambda}\right) \neq 0$. Then $J_{\lambda}^{i} E_{\lambda}$ is a totally isotropic subbundle of $E$ of non-zero rank.

P147-GilkeyIvanova-v3a: submitted to World Scientific on October 26, 20184 
Proof: Assume that $E$ and $J$ satisfy the hypothesis of the Lemma. Set:

$$
E_{\lambda, i}:=J_{\lambda}^{i}\left(E_{\lambda}\right) .
$$

Since $J$ has constant Jordan normal form, $E_{\lambda, i}$ is a smooth vector bundle over $M$. Fix a point $P$ of $M$ and let $v_{1}$ and $v_{2}$ be vectors in the fiber $E_{\lambda, i}(P)$. There exist vectors $w_{1}, w_{2} \in E_{\lambda, i}(P)$ so $v_{1}=J_{\lambda}^{i} w_{1}$ and $v_{2}=J_{\lambda}^{i} w_{2}$. Note that $2 i \geq i+1$, that $J_{\lambda}$ is self-adjoint, and that $J_{\lambda}^{2 i}=0$ on $E_{\lambda}$. We demonstrate that $E_{\lambda, i}$ is totally isotropic and thereby complete the proof by computing:

$$
\left(v_{1}, v_{2}\right)=\left(J_{\lambda}^{i} w_{1}, J_{\lambda}^{i} w_{2}\right)=\left(J_{\lambda}^{2 i} w_{1}, w_{2}\right)=0 .
$$

\section{$3 \quad$ Bundles over projective space}

Let $V$ be a vector space of signature $(p, q)$. We decompose $V=V^{+} \oplus V^{-}$as an orthogonal direct sum, where $V^{+}$is a maximal spacelike subspace of dimension $q$ and $V^{-}$is the complementary maximal timelike subspace of dimension $p$. Let $\mathbb{P}\left(V^{+}\right)$be the projective space of lines in $V^{+}$. We define trivial bundles over $\mathbb{P}\left(V^{+}\right)$by setting:

$$
\begin{aligned}
& \mathbb{V}^{+}:=\mathbb{P}\left(V^{+}\right) \times V^{+}, \\
& \mathbb{V}^{-}:=\mathbb{P}\left(V^{+}\right) \times V^{-}, \text {and } \\
& \mathbb{V}:=\mathbb{V}^{+} \oplus \mathbb{V}^{-}=\mathbb{P}\left(V^{+}\right) \times V .
\end{aligned}
$$

These bundles inherit natural inner products from the given inner product on $V$. Let $\langle x\rangle:=x \cdot \mathbb{R}$ be the line thru an element $x \in S\left(V^{+}\right)$. The classifying line bundle $\gamma$ and the orthogonal complement $\gamma^{\perp}$ over $\mathbb{P}\left(V^{+}\right)$are the subbundles of $\mathbb{V}^{+}$defined by:

$$
\begin{aligned}
& \gamma:=\left\{(\langle x\rangle, y) \in \mathbb{P}\left(V^{+}\right) \times V^{+}: y \in\langle x\rangle\right\}, \text { and } \\
& \gamma^{\perp}:=\left\{(\langle x\rangle, y) \in \mathbb{P}\left(V^{+}\right) \times V^{+}: y \perp\langle x\rangle\right\} .
\end{aligned}
$$

Note that $\gamma_{\langle x\rangle}=\langle x\rangle$, i.e. the fiber of $\gamma$ over an element $\langle x\rangle$ of $\mathbb{P}\left(V^{+}\right)$is just the line $\langle x\rangle$ itself. For that reason $\gamma$ has also been called the tautological line bundle. This bundle plays an important role in the classification of real line bundles and in our further considerations. In the following Lemma, we compare non-trivial (i.e. positive rank) subbundles of $\gamma^{\perp}$ and $\mathbb{V}^{-}$.

Lemma 3.1 Let $V=V^{+} \oplus V^{-}$be a vector space of signature $(p, q)$, where $p<q$. Let $\mathbb{V}^{-}$and $\gamma^{\perp}$ be the bundles over $\mathbb{P}\left(V^{+}\right)$defined in equations (3.1) and (3.2), respectively. Then no non-trivial subbundle of $\mathbb{V}^{-}$is isomorphic to a non-trivial subbundle of $\gamma^{\perp}$.

P147-GilkeyIvanova-v3a: submitted to World Scientific on October 26, 20185 
Proof: The Stiefel-Whitney classes 12 are cohomological invariants of vector bundles. Let $w_{1}:=w_{1}(\gamma)$ be the first Stiefel-Whitney class of the classifying line bundle $\gamma$. The cohomplogy ring of the projective space $\mathbb{P}\left(V^{+}\right)$is the truncated polynomial ring12:

$$
H^{*}\left(\mathbb{P}\left(V^{+}\right) ; \mathbb{Z}_{2}\right)=\mathbb{Z}_{2}\left[w_{1}\right] /\left(w_{1}^{q}=0\right) .
$$

To prove Lemma 3.1, we suppose the contrary, i.e. that there exist positive rank subbundles $E_{1}$ of $\gamma^{\perp}$ and $E_{2}$ of $\mathbb{V}^{-}$so that $E_{1}$ is isomorphic to $E_{2}$, and argue for a contradiction. Let $r=\operatorname{rank}\left(E_{1}\right)$. Since $E_{1} \subset \gamma^{\perp}$, we may use Lemma 4.4.49 to see that $w_{r}\left(E_{1}\right)=w_{1}^{r}$. Furthermore, as $E_{1}$ is isomorphic to $E_{2}$, we may conclude $w_{r}\left(E_{2}\right)=w_{1}^{r}$. As $\mathbb{V}^{-}=E_{2} \oplus E_{2}^{\perp}$, we have the following factorization

$$
1=w\left(E_{2}\right) w\left(E_{2}^{\perp}\right)=\left(1+\ldots+w_{1}^{r}\right) w\left(E_{2}^{\perp}\right) \quad \text { in } \quad H^{*}\left(\mathbb{P}\left(V^{+}\right) ; \mathbb{Z}_{2}\right) .
$$

Since rank $E_{2}=p-r<q$, the truncation $\left(w_{1}^{q}=0\right)$ in $H^{*}\left(\mathbb{P}\left(V^{+}\right) ; \mathbb{Z}_{2}\right)$ plays no role, so we have the factorization

$$
1=\left(1+\ldots+w_{1}^{r}\right) w\left(E_{2}^{\perp}\right) \quad \text { in } \quad \mathbb{Z}_{2}\left[w_{1}\right],
$$

which is impossible.

We use Lemma 3.1 to establish the following Lemma:

Lemma 3.2 Let $V=V^{+} \oplus V^{-}$be a vector space of signature $(p, q)$, where $p<q$. Let $\mathbb{V}^{-}$and $\gamma^{\perp}$ be the bundles over $\mathbb{P}\left(V^{+}\right)$defined in equations (3.1) and (3.2), respectively. There is no totally isotropic non-trivial subbundle of $\gamma^{\perp} \oplus \mathbb{V}^{-}$.

Proof: We suppose, to the contrary, that there exists a totally isotropic nontrivial subbundle $E$ of $\gamma^{\perp} \oplus \mathbb{V}^{-}$. Let $\pi^{+}$be orthogonal projection on $\gamma^{\perp}$ and let $\pi^{-}$be orthogonal projection on $\mathbb{V}^{-}$. Set

$$
E^{+}:=\pi^{+}(E) \subset \gamma^{\perp} \quad \text { and } \quad E^{-}:=\pi^{-}(E) \subset \mathbb{V}^{-} .
$$

Note that $\operatorname{ker} \pi^{+}=\mathbb{V}^{-}$and $\operatorname{ker} \pi^{-}=\gamma^{\perp}$. As $E$ is totally isotropic, every vector in $E$ is null. Thus

$$
E \cap \mathbb{V}^{-}=E \cap \gamma^{\perp}=\{0\} .
$$

Consequently, $\pi^{+}$and $\pi^{-}$define isomorphisms between $E$ and $E^{+}$and between $E$ and $E^{-}$, respectively. Thus $E^{+}$, which is a non-trivial subbundle of $\gamma^{+}$, is isomorphic to $E^{-}$, which is a non-trivial subbundle of $\mathbb{V}^{-}$. This contradicts Lemma 3.1 .

P147-GilkeyIvanova-v3a: submitted to World Scientific on October 26, 20186 


\section{Proof of Theorem 1.2}

Let $V=V^{+} \oplus V^{-}$be a vector space of signature $(p, q)$, where $p<q$, and where $V^{+}$and $V^{-}$are orthogonal maximal spacelike and timelike subspaces of $V$, respectively. Let $R$ be a spacelike Jordan Osserman algebraic curvature tensor on $V$. Let $x \in S^{+}(V)$. Since $\mathcal{J}_{R}(x)$ is self-adjoint and since $\mathcal{J}_{R}(x) x=0$, $\mathcal{J}_{R}(x)$ preserves the orthogonal complement $x^{\perp}$; we let $\tilde{\mathcal{J}}_{R}(x)$ denote the restriction of $\mathcal{J}_{R}(x)$ to $x^{\perp}$; this is often called the reduced Jacobi operator. The Jacobi operator can be represented in the form

$$
\mathcal{J}_{R}(x)=\left(\begin{array}{cc}
0 & 0 \\
0 & \tilde{\mathcal{J}}_{R}(x)
\end{array}\right) \quad \text { on } \quad x \oplus x^{\perp} .
$$

Thus to prove Theorem 1.2, it suffices to show that $\tilde{\mathcal{J}}_{R}(x)$ is diagonalizable.

If $\lambda \in \mathbb{C}$, then let $\tilde{J}_{\lambda}$ and $E_{\lambda}$ be defined by $\tilde{\mathcal{J}}_{R}$ using equations (2.1) and (2.2), respectively. We may then use Lemma 2.1 to decompose

$$
\gamma^{\perp} \oplus \mathbb{V}^{-}=\oplus_{\Im(\lambda) \geq 0} E_{\lambda} \text { over } \mathbb{P}\left(V^{+}\right)
$$

where the induced metric on each eigenbundle $E_{\lambda}$ is non-degenerate. By Lemma 3.2, $E_{\lambda}$ does not contain a totally isotropic subbundle. Thus, by Lemma 2.3, $\tilde{\mathcal{J}}_{\lambda}=0$ on $E_{\lambda}$. Consequently $\tilde{\mathcal{J}}_{R}$ is diagonalizable on $E_{\lambda}$ if $\lambda \in \mathbb{R}$.

To complete the proof, we must show that all the eigenvalues are real. Suppose, to the contrary, that there exists an eigenvalue $\lambda$ of $\tilde{\mathcal{J}}_{R}$ so that $\Im(\lambda) \neq 0$; we argue for a contradiction. By Lemma 2.2, $E_{\lambda}=E_{\lambda}^{+} \oplus E_{\lambda}^{-}$ decomposes as the orthogonal direct sum of maximal spacelike and timelike subbundles. We define a bundle map $\mathcal{I}$ of $E_{\lambda}$ by setting:

$$
\mathcal{I}:=\frac{\tilde{\mathcal{J}}_{R}-\Re(\lambda) \mathrm{Id}}{\Im(\lambda)} .
$$

The definition of $\tilde{\mathcal{J}}_{\lambda}$ given in (2.1) and the fact that $\tilde{\mathcal{J}}_{\lambda}=0$ on $E_{\lambda}$ then imply that $\mathcal{I}^{2}=-$ id on $E_{\lambda}$. Since $\mathcal{I}$ is self-adjoint, $\mathcal{I}$ is a para-isometry of $E_{\lambda}$ that interchanges the roles of spacelike and timelike vectors. Thus, $\mathcal{I}$ defines an isomorphism between $E_{\lambda}^{+}$and $E_{\lambda}^{-}$.

Let $\pi^{+}$and $\pi^{-}$be orthogonal projections on $\gamma^{\perp}$ and $\mathbb{V}^{-}$, respectively. Since $E_{\lambda}^{+}$contains no timelike vectors and since $\operatorname{ker}\left(\pi^{+}\right)=\mathbb{V}^{-}$is timelike, $\operatorname{ker} \pi^{+} \cap E_{\lambda}^{+}=\{0\}$ and $\pi^{+}$is an isomorphism from $E_{\lambda}^{+}$to $\pi^{+}\left(E_{\lambda}\right)$. Similarly, $\pi^{-}$is an isomorphism from $E_{\lambda}^{-}$to $\pi^{-}\left(E_{\lambda}\right)$. Thus $\pi^{+}\left(E_{\lambda}\right)$, which is a non-trivial subbundle of $\gamma^{\perp}$, is isomorphic to $\pi^{-}\left(E_{\lambda}^{-}\right)$, that is a non-trivial subbundle of $\mathbb{V}^{-}$. This contradicts Lemma 3.1.

P147-GilkeyIvanova-v3a: submitted to World Scientific on October 26, 20187 


\section{Acknowledgments}

Research of both authors supported in part by the NSF (USA) and MPI (Leipzig).

\section{References}

1. N. Blažić, N. Bokan and P. Gilkey, A Note on Osserman Lorentzian manifolds, Bull. London Math. Soc. 29 (1997), 227-230.

2. N. Blažić, N. Bokan, P. Gilkey and Z. Rakić,Pseudo-Riemannian Osserman manifolds, J. Balkan Soc. Geometers 12 (1997), 1-12.

3. A. Bonome, R. Castro, E. García-Río, L. Hervella, R. Vázquez-Lorenzo, Nonsymmetric Osserman indefinite Kähler manifolds, Proc. Amer. Math. Soc. 126 (1998), 2763-2769.

4. Q.-S. Chi, A curvature characterization of certain locally rank-one symmetric spaces, J. Differential Geom. 28 (1988), 187-202.

5. I. Dotti and M. Druetta, Negatively curved homogeneous Osserman spaces, Differential Geom. Appl. 11 (1999), 163-178.

6. E. García-Rió, D. Kupeli and M. E. Vázquez-Abal, On a problem of Osserman in Lorentzian geometry, Differential Geom. Appl. 7 (1997), 85-100.

7. E. García-Rio, D. N. Kupeli, and R. Vázquez-Lorenzo Osserman manifolds in semi-Riemannian geometry, Lecture notes in Mathematics, Springer Verlag, to appear.

8. E. García-Rió, M. E. Vázquez-Abal and R. Vázquez-Lorenzo, Nonsymmetric Osserman pseudo-Riemannian manifolds, Proc. Amer. Math. Soc. 126 (1998), 2771-2778.

9. P. Gilkey, Natural Operators Defined by the Riemann Curvature Tensor, World Scientific Press, ISBN 981-02-4752.

10. P. Gilkey and R. Ivanova, The Jordan normal form of Osserman algebraic curvature tensors, preprint.

11. P. Gilkey, A. Swann, and L. Vanhecke, Isoparametric geodesic spheres and a conjecture of Osserman regarding the Jacobi operator, Quart. J. Math. Oxford Ser. 46 (1995), 299-320.

12. J. Mil.nor and J. Stasheff, Characteristic Classes, Annals of Math. Studies, Princeton University Press (1974).

13. R. Osserman, Curvature in the eighties, Amer. Math. Monthly 97 (1990), 731-756. 